\title{
THE ENDOMORPHISM SEMIGROUP OF AN ENDOMAPPING OF A FINITE SET
}

\author{
Subrata Majumdar, *Mohd. Altab Hossain and Kalyan Kumar Dey \\ Department of Mathematics \\ University of Rajshahi, Rajshai-6205, Bangladesh. \\ *Email: al_math_bd@yahoo.com
}

Received 29.06.2011

Accepted 22.10.2011

\begin{abstract}
The structure of the semigroup of all endomorphisms of an endomapping of a finite set has been determined. This has been done by naturally representing the endomapping by a directed graph, and determining the structure of the endomorphism semigroup of this graph.
\end{abstract}

Keywords: Endomorphism semigroup, Transformation semigroup, Full transformation semigroup, Direct product, Wreath product, Directed graph.

\section{Introduction}

Let $X$ be a finite non-epmty set and let $f: X \rightarrow X$ be an endomapping of $X$. The set of all endomappings of $X$ is a semigroup under the composition of maps and is called the full transformation semigroup on $X$ and is denoted by $E(X)$. If the number of elements of $X$ is $n$, we shall also write $F_{n}$ for $E(X)$. A map $g: X \rightarrow X$ is called an endomorphism of $f$ if $g f=f g$ i.e., if g belongs to the centraliser of $f$ in $E(X)$. The centraliser of $f, C(f)=\{g \in E(X) \mid g f=f g\}$, is a transformation semigroup on $X$ and is called the endomorphism semigroup of $f$. We denote this semigroup by End $f$. In this paper, we shall determine the structure of this semigroup End $f$ for a class of endomappings $f$ such that, for each $x \in X$, there exists a positive integer $r_{x}$ with the property that $f^{r_{x}+1}(x)=f^{r_{x}}(x)$. The technique of structure-determination consists of

(i) representing $f$ by a directed graph $G(f)$ with vertices the points of $X$ and edges $x \rightarrow f(x)$, and

(ii) determining the structure of the semigroup $\operatorname{End}(G(f))$ of those transformations $T$ of this directed graph $G(f)$ such that $T(f(x))=f(T(x))$ i.e., $T(x \rightarrow f(x))=(T(x) \rightarrow T(f(x)))$.

Since $T$ maps vertices onto vertices and edges onto corresponding edges, $T$ is called an endomorphism of the digraph of $f$. If $\mathrm{g}$ is the endomapping of $X$ 
induced by $T$, the map $g \rightarrow T$ is an isomorphism of End $f$ into the endomorphism semigroup of $G(f)$. Then $G(f)$ has an appearance of the type:

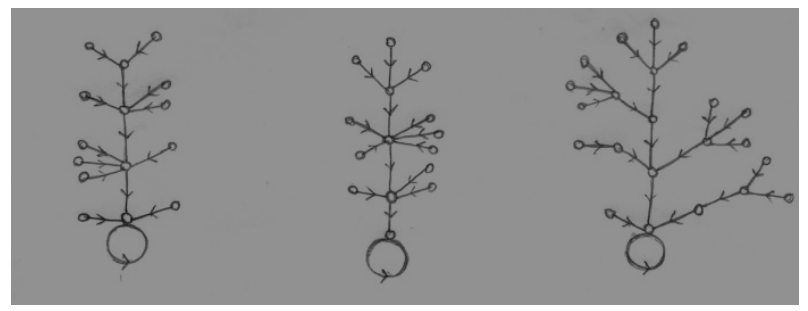

Fig.1

We shall determine the structure of the transformation semigroup End $(G(X))$ for a class of endomappings $f$. The discussion in the above ensures that the structure of End $f$ will be known through the isomorphism End $f \cong$ End $(G(X))$.

\section{Necessary Preliminaries}

To determine the structure of the transformation semigroup $\operatorname{End}(G(X))$ we need some results of [4] about the direct product and wreath product of transformation semigroups. We recall these in the following:

A semigroup $S$ is called a transformation semigroup on a nonempty set $\mathrm{X}$ and is written $(S, X)$ if there is a map $S \times X \rightarrow X$ given by $(s, x) \rightarrow x$ such that $\left(s_{1} s_{2}\right)(x)=s_{1}\left(s_{2}(x)\right)$. If $\mathrm{S}$ is a monoid, then $1(x)=x$, for each $x \in X$. For transformation semigroups $S_{1}$ and $S_{2}$ on disjoint non-empty sets $X_{1}, X_{2}$, the direct product $S_{1} \times S_{2}$ is a transformation semigroup on $X_{1} \cup X_{2}$ with action given by $\left(s_{1}, s_{2}\right)\left(x_{1}\right)=s_{1}\left(x_{1}\right)$ and $\left(s_{1}, s_{2}\right)\left(x_{2}\right)=s_{2}\left(x_{2}\right)$.

For two non-empty sets $X_{1}, X_{2}$, the wreath product $S_{1} \varsigma S_{2}$ is a transformation semigroup on $X_{1} \times X_{2}$ and consists of maps $\theta: X_{1}, X_{2} \rightarrow X_{1}, X_{2}$ given by $\theta\left(x_{1}, x_{2}\right)=\left(s_{1, x_{2}}\left(x_{1}\right), s_{2}\left(x_{2}\right)\right), s_{1, x_{2}}$ being an element of $S_{1}$ determined by $x_{2}$.

The following results in [4] show that (i) wreath product has a description in terms of direct product which makes the sense that wreath product is associative and is distributive over direct product.

\section{Theorem 2.1}

$$
\left(S_{1} \varsigma S_{2}, X \times X_{2}\right) \cong\left(\left(\underset{x_{2} \in X_{2}}{\times} S_{1, x_{2}}\right) \times S_{2},\left(\underset{x_{2 \in X_{2}}}{\bigcup} X_{1, x_{2}}\right) \times X_{2}\right)
$$

where each $x_{2} \in X_{2}, S_{1, x_{2}} \cong S_{1}$ and $\left|X_{1, x_{2}}\right|=\left|X_{1}\right|$. 
Theorem $2.2\left(\left(S_{1} \varsigma S_{2}\right) \varsigma S_{3},\left(X_{1} \times X_{2}\right) \times X_{3}\right) \cong\left(S_{1} \varsigma\left(S_{2} \varsigma S_{3}\right), X_{1} \times\left(X_{2} \times X_{3}\right)\right)$.

Theorem 2.3

$\left(S_{1} \varsigma\left(S_{2} \times S_{3}\right), X_{1} \times\left(X_{2} \cup X_{3}\right)\right) \cong\left(\left(S_{1} \varsigma S_{2}\right) \times\left(S_{1} \varsigma S_{3}\right),\left(X_{1} \times X_{2}\right) \cup\left(X_{1} \times X_{3}\right)\right)$.

Remarks.

(i) If $S_{2}=\left\{1_{X_{2}}\right\}$, then $\left(S_{1} \times S_{2}, X_{1} \cup X_{2}\right)$ may be identified with $\left(S_{1}, X_{1}\right)$ and $\left(S_{1} \varsigma S_{2}, X_{1} \times X_{2}\right)$ with $\left(\prod_{x_{2} \in X_{2}} S_{1, x_{2}}, \bigcup_{x_{2} \in X_{2}} X_{1, x_{2}}\right)$ where $\left|X_{1, x_{2}}\right|=\left|X_{1}\right|$.

(ii) If $S_{1}=\left\{1_{X_{1}}\right\}$, then both $\left(S_{1} \times S_{2}, X_{1} \cup X_{2}\right)$ and $\left(S_{1} \varsigma S_{2}, X_{1} \times X_{2}\right)$ may be identified with $\left(S_{2}, X_{2}\right)$.

(iii) If $X_{1}=X_{2}=X$, then $\left(S_{1} \varsigma S_{2}, X \times X\right)$ may be identified with $\left.\left(\prod_{x \in X} S_{1, x}\right) \times S_{2}, \bigcup_{x \in X} X_{1, x} \cup X\right)$. As semigroups, $S_{1} \varsigma S_{2} \cong\left(\prod_{x \in X} S_{1, x}\right) \times S_{2}$.

\section{Structure of the endomorphism semigroup End $f$}

We now determine the structure of End $f$ through representation of $f$ by a directed graph. We begin with the following lemma:

Lemma 3.1 Let $G(f)$, the directed graph of $f$, be given by:

Then End $f \cong E(m)=\left\{\sigma_{0}, \sigma_{1}, \sigma_{1}^{2} \cdots \cdots, \sigma_{1}^{m}\right\}$, is a cyclic semigroup generated by $\sigma_{1}$ and adjoined with an identity element of $\sigma_{0}$, with $\sigma_{1}^{m}$ as the zero element, i.e., $\sigma_{1}^{m} \sigma_{1}^{i}=\sigma_{1}^{m}, \quad 0 \leq i \leq m$.

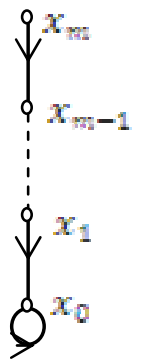

Fig. 2

Proof. We observe that $g \in$ End $f$ if and only if (i) $g\left(x_{0}\right)=x_{0}$ and if (ii) $\mathrm{r}$ is the positive integer such that $g=1_{X}$ or $g\left(x_{r}\right)=x_{0}$, then $g\left(x_{i}\right)=x_{0}$, for each $i \leq r$, and $g\left(x_{s}\right)=x_{s-1} \quad$ for $\quad r+1 \leq s \leq m$. We write $\overline{\sigma_{0}}=\left(\begin{array}{ccc}x_{0} & x_{1} & \cdots \cdots x_{m} \\ x_{0} & x_{1} & \cdots \cdots x_{m}\end{array}\right)$ and $\overline{\sigma_{i}}=\left(\begin{array}{ccccc}x_{0} & x_{1} & \cdots \cdots x_{i} & x_{i+1} \cdots \cdots x_{m} \\ x_{0} & x_{0} & \cdots \cdots x_{0} & x_{1} \cdots \cdots x_{m-1}\end{array}\right), \quad 1 \leq i \leq m$. 
Then End $f=\overline{E(m)}=\left\{\overline{\sigma_{0}}, \overline{\sigma_{1}}, \cdots \cdots, \overline{\sigma_{m}}\right\}$ with multiplication given by $\overline{\sigma_{0}} \overline{\sigma_{r}}=\overline{\sigma_{r}} \overline{\sigma_{0}}=\overline{\sigma_{r}}$ for $0 \leq r \leq m, \quad \overline{\sigma_{i}} \overline{\sigma_{j}}=\overline{\sigma_{j}} \overline{\sigma_{i}}=\overline{\sigma_{i+j}} \quad$ for $0<i, j<m$ and $i+j \leq m$, and $\overline{\sigma_{r}} \overline{\sigma_{m}}=\overline{\sigma_{m}} \overline{\sigma_{r}}=\overline{\sigma_{m}}$ for $0 \leq r \leq m$. Then it is clear that $\overline{E(m)} \cong E(m)$ and hence statement of the lemma is alright.

We next consider the following situation:

Lemma 3.2 Let $f$ be given by the directed graph $G(f)$ in fig.3:

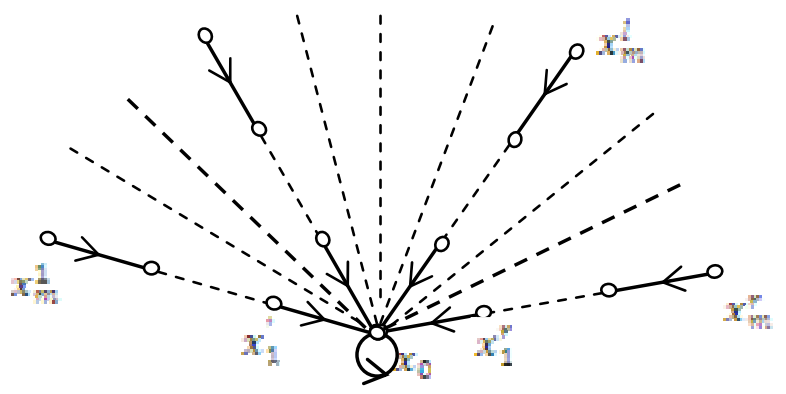

Fig.3

consisting of $r$ directed subgraphs each being a chain of length $m$ and each with the loops at $x_{0}$. Then End $f \cong E(m) \varsigma F_{r}$

Here, as mentioned earlier, $F_{r}$ is the full transformation semigroup on a set with $r$ elements.

Proof. Since each $g \in$ End $f$ must map $x_{0}$ onto itself and since each maximal chain ending at $x_{0}$ has the same length $\mathrm{m}$, End $f$ may be identified with the semigroup of all endomorphisms of an endomapping $f^{\prime}$ of $X$ whose directed graph is $C_{m} \times\{1,2, \cdots \cdots, r\}, C_{m}$ being the directed graph given by the figure in Lemma 3.3. It therefore follows from the mentioned lemma and definition of wreath product that End $f \cong E(m) \varsigma F_{r}$.

We now observe that if $f$ is given by the directed graph $G(f)$ in fig.4:

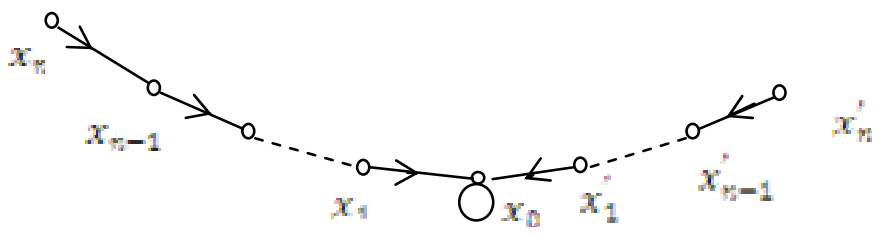

Fig.4

with $\mathrm{m}>\mathrm{n}$, then 


$$
\left\{\begin{aligned}
\text { End } f= & \left(\text { End } f_{1} \times \text { End } f_{2}\right) \cup\left(\text { End } f_{1} \times \operatorname{Hom}\left(T_{2}, T_{1}\right)\right) \cup \\
& \left(\operatorname{Hom}\left(T_{1}, T_{2}\right) \times \operatorname{Hom}\left(T_{1} \times T_{2}\right)\right) \cup\left(\operatorname{Hom}\left(T_{1}, T_{2}\right), \text { End } f_{2}\right),
\end{aligned}\right.
$$

where $f_{1}$ and $f_{2}$ are $\mathrm{f}$ restricted to $\left\{x_{0}, x_{1}, \cdots \cdots, x_{m}\right\}$ and $\left\{x_{0}, \quad x_{1}^{\prime}, \cdots \cdots, \quad x_{n}^{\prime}\right\}$ respectively and $\operatorname{Hom}\left(T_{i}, T_{j}\right) \quad(i, j=1,2 ; i \neq j)$ denotes the set of maps the directed graph $T_{i}$ into the directed graph $T_{j}$ of $f_{i}$ and $f_{j}$ respectively.

Here

$$
\left\{\begin{array}{l}
\left(\operatorname{End} T_{i}\right) \operatorname{Hom}\left(T_{j}, T_{i}\right) \subseteq \operatorname{Hom}\left(T_{j}, T_{i}\right) \\
\operatorname{Hom}\left(T_{i}, T_{j}\right) \operatorname{Hom}\left(T_{j}, T_{i}\right) \subseteq \operatorname{End} T_{j}
\end{array}\right.
$$

Also, if $\phi=\left(\begin{array}{ccccccc}x_{0} & x_{1} & \cdots \cdots & x_{m-n} & x_{m-n+1} & \cdots & x_{n} \\ x_{0} & x_{0} & \cdots \cdots & x_{0} & x_{1}^{\prime} & \cdots \cdots & x_{n}^{\prime}\end{array}\right)$ and $\varphi=\left(\begin{array}{cccc}x_{0} & x_{1}^{\prime} & \cdots \cdots & x_{n}^{\prime} \\ x_{0} & x_{1} & \cdots \cdots & x_{n}\end{array}\right)$, then it is easy to see that

$$
\left\{\begin{array}{l}
\operatorname{Hom}\left(T_{1}, T_{2}\right)=\left(\text { End } T_{2}\right) \phi \\
\operatorname{Hom}\left(T_{2}, T_{1}\right)=\left(\text { End } T_{1}\right) \varphi
\end{array}\right.
$$

It follows from the above facts that

$$
\left\{\begin{array}{l}
\left(\text { End } f_{1}\right) \times \operatorname{Hom}\left(T_{2}, T_{1}\right)=\left(\text { End } f_{1}\right)^{2} \varphi, \\
\left(\operatorname{Hom}\left(T_{1}, T_{2}\right)\left(\text { End } f_{1}\right)=\left(\text { End } f_{2}\right) \phi\left(\text { End } f_{1}\right),\right. \\
\left(\operatorname{End} f_{2}\right) \times \operatorname{Hom}\left(T_{1}, T_{2}\right)=\left(\text { End } f_{2}\right)^{2} \varphi, \\
\left(\operatorname{Hom}\left(T_{2}, T_{1}\right)\left(\text { End } f_{2}\right)=\left(\text { End } f_{1}\right) \varphi\left(\text { End } f_{2}\right),\right. \\
\left(\operatorname{Hom}\left(T_{1}, T_{2}\right)\right)\left(\operatorname{Hom}\left(T_{2}, T_{1}\right)\right)=\left(\text { End } f_{2}\right) \phi\left(\text { End } f_{1}\right) \varphi, \\
\left(\operatorname{Hom}\left(T_{2}, T_{1}\right)\right)\left(\operatorname{Hom}\left(T_{1}, T_{2}\right)\right)=\left(\text { End } f_{1}\right) \varphi\left(\text { End } f_{2}\right) \phi .
\end{array}\right.
$$

We therefore have proved that.

Lemma 3.3 The semigroup-structure of End $f$ of $\mathrm{f}$ given by fig.4 is completely given by the expressions from (1) to (5).

We now consider the following situation:

Let $T_{1, k_{1}}^{\prime}, \cdots, T_{r_{1}, k_{1}}^{\prime}$ represent $r_{1}$ chains, each of length $k_{1}$ and each with a loop at the same point $x_{0}$. Let $\mathrm{T}$ denote the directed graph consisting of all such $T_{1, k_{j}}^{\prime}, \cdots, T_{r_{j}, k_{j}}^{\prime}, 1 \leq j \leq n$. Let $f$ be given by the directed graph $G(f)=T$. Then $G(f)=T$ will look like fig.5. 


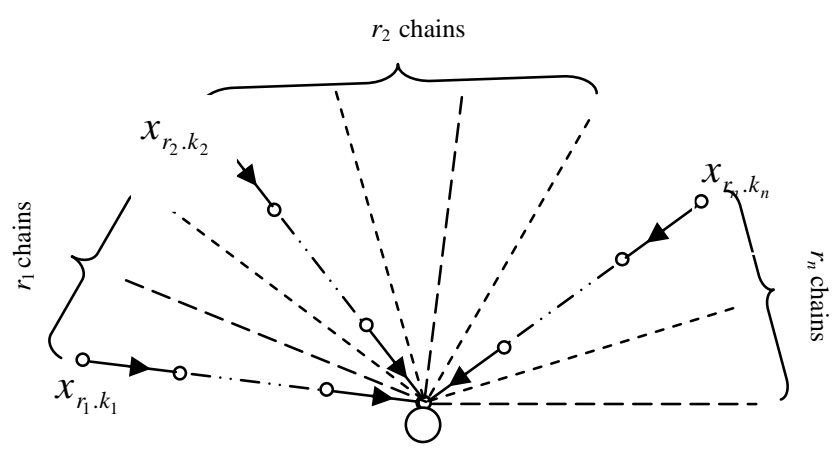

Fig. 3.

Theorem 3.1 Let $f$ be given by the directed graph $G(f)$ as in fig.5 with $f^{k}(X)$ a singleton, where $k=\max \left\{k_{1}, \cdots, k_{m}\right\}$. Then

$$
\text { End } f=\bigcup\left[\text { End } T^{i_{1}} \times \cdots \cdots \times \text { End } T^{i_{u}} \times\left(\times_{u<v, v^{\prime}<k}^{v \neq v^{\prime}} \operatorname{Hom}\left(T^{i_{v}}, T^{i_{v^{\prime}}}\right)\right)\right] \text {, }
$$

the union being taken over all permutations $\left(\begin{array}{cccc}1 & 2 & 3 & \cdots \cdots k \\ i_{1} & i_{2} & i_{3} & \cdots \cdots \\ i_{k}\end{array}\right)$.

Here,

$$
\left\{\begin{array}{l}
\operatorname{End} T^{i}=\operatorname{End~}^{i, \alpha} \varsigma F_{i_{r}} \quad\left(\alpha \in\left\{1, \quad 2, \quad 3, \cdots \cdots, \quad i_{r}\right\}\right), \\
\operatorname{Hom}\left(T^{i_{v}}, T^{i_{v^{\prime}}}\right)=\times_{1 \leq \beta \leq r_{i_{v^{\prime}}}}^{1 \leq \alpha \leq r_{i^{\prime}}} \operatorname{Hom}\left(T_{\alpha}^{i_{v}}, T_{\beta}^{i_{v^{\prime}}}\right) .
\end{array}\right.
$$

The products of End $T^{i, \alpha}$ with themselves and with $\operatorname{Hom}\left(T_{\alpha}^{i}, T_{\beta}^{j}\right)$ as well as the products of $\operatorname{Hom}\left(T_{\alpha}^{i}, T_{\beta}^{j}\right)$ among themselves are given by (5). Also, the End $T^{i, \alpha}$, s are isomorphic to one another, since $T^{i, \alpha}$, s are chains of the same length.

Proof. The proof is exactly similar to that of lemma 3.3.

\section{Conclusion}

In the most general case of an endomapping, the directed graph representing the endomapping consists of a finite number of disjoint directed rooted trees. In this case, the method of determining the endomorphism semigroup of this endomapping will be almost similar but complicated. It will be taken up in future. 


\section{REFERENCES}

1. Cliford, A. H. and Preston, G. B. Algebraic theory of semigroups, American Mathematical. Society, New York, 1961.

2. Hall Jr., M. The theory of groups, Macmillan Co., New York, 1964.

3. MacLane, S. and Birkhoff, G. Algebra, MacMillan, New York, 2nd ed., 1979.

4. Majumdar, S., Dey, K.K. and Hossain, M. A. Direct product and wreath product of transformation semigroups, accepted for publication to GANIT- a Journal of Bangladesh Mathematical Society, 2011.

5. Meldrum, J. D. P. Wreath products of groups and semigroups, Longman, 1995. 